Pacific Journal of Mathematics

DIVISION OF DISTRIBUTIONS 


\title{
DIVISION OF DISTRIBUTIONS
}

\author{
Elemer E. Rosinger
}

This paper deals with division in an associative commutative algebra containing the distributions in $\mathbf{R}^{\mathbf{n}}$.

1. Introduction. In [5] and [6], a family $\left(A_{p, \lambda} \mid p \in \bar{N}^{n}, \lambda \in \Lambda\right)$ of associative, commutative algebras with unit element were constructed, with the following main properties:

(1) $\mathscr{D}^{\prime}\left(R^{n}\right) \subset A_{p, \lambda}, \forall p \in \bar{N}^{n}, \lambda \in \Lambda$, (here, $N=\{0,1,2, \cdots\}, \bar{N}=N \cup\{\infty\}$ and $n \in N, n \geqq 1$ );

(2) The multiplication in each of the algebras $A_{p, \lambda}, p \in \bar{N}^{n}, \lambda \in \Lambda$, induces on $\mathscr{C}^{\infty}\left(R^{n}\right)$ the usual multiplication of functions and the function $\psi \in \mathscr{C}^{\infty}\left(R^{n}\right)$, with $\psi(x)=1, \forall x \in R^{n}$, is the unit element in the algebras;

(3) for each $\lambda \in \Lambda$, there exist linear mappings $D^{p}: A_{q+p, \lambda} \rightarrow A_{q, \lambda}$, with $p \in N^{n}, q \in \bar{N}^{n}$, such that

(3.1) $D^{p}$ satisfies on $A_{q+p, \lambda}$ the Leibnitz rule of product derivative.

(3.2) $D^{p}$ is the usual distribution derivative on $\mathscr{C}^{\infty}\left(R^{n}\right) \oplus \mathscr{D}_{\delta}^{\prime}\left(R^{n}\right)$, where $\mathscr{D}_{\delta}^{\prime}\left(R^{n}\right)=\left\{S \in \mathscr{D}^{\prime}\left(R^{n}\right) \mid \operatorname{supp} S\right.$ is finite\};

(4) The following relations hold for the Dirac $\delta_{x_{0}}$ distribution, concentrated in $x_{0} \in R^{n}$ :

$$
\left(x-x_{0}\right)^{r} \cdot D^{q} \delta_{x_{0}}=0 \in A_{p, \lambda}, \quad \forall p \in N^{n}, \quad \lambda \in \Lambda,
$$

if $q, r \in N^{n}, r \geqq p+e, r \geqq q+e$, where $e=(1, \cdots, 1) \in N^{n}$.

In the present paper, within the one dimensional case $n=1$, necessary or sufficient conditions are given for $T \in A_{p, \lambda}$, in order to be a solution of one of the equations $x^{m} \cdot T=0 \in A_{p, \lambda}$ and $x^{m} \cdot T=S \in A_{p, \lambda}$, with $m \in N, m \geqq 1$.

2. Notations. Several classes of sequences of complex valued smooth functions (see [5] and [6]) will be needed.

(1) $\mathscr{W}=N \rightarrow \mathscr{C}^{\infty}\left(R^{1}\right)$; if $s \in \mathscr{W}, \quad \nu \in N, \quad x \in R^{1}$, then $s(\nu) \in$ $\mathscr{C}^{\infty}\left(R^{1}\right), s(\nu)(x) \in C^{1}$; for $\psi \in \mathscr{C}^{\infty}\left(R^{1}\right)$ denote $u(\psi) \in \mathscr{W}$, where $u(\psi)(\nu)$ $=\psi, \forall \nu \in N ; \mathscr{W}$ is in a natural way an associative, commutative algebra (the vector spaces and algebras are considered over the field $C^{1}$ of 
complex numbers), with the unit element $u(1)$ and zero element $u(0)$; thus, $\mathcal{O}=\{u(0)\}$ is the null space in $\mathscr{W}$;

(2) $D: \mathscr{W} \rightarrow \mathscr{W}$ is defined by $(D s)(\nu)(x)=(D s(\nu))(x), \forall s \in \mathscr{W}$, $\nu \in N, x \in R^{1}$; for given $x_{0} \in R^{1}$, define $\tau_{x_{0}}: \mathscr{W} \rightarrow \mathscr{W}$ by $\left(\tau_{x_{0}} s\right)(\nu)(x)=$ $s(\nu)\left(x-x_{0}\right), \forall s \in \mathscr{W}, \nu \in N, x \in R^{1}$;

(3) $\quad U=\left\{u(\psi) \mid \psi \in \mathscr{C}^{\infty}\left(R^{1}\right)\right\}$;

(4) $\mathscr{S}_{0}$ is the set of $s \in \mathscr{W}$, weakly convergent in $\mathscr{D}^{\prime}\left(R^{1}\right) ; \mathscr{V}_{0}$ is the kernel of the linear surjection:

$$
\mathscr{S}_{0} \ni s \rightarrow\langle s, \cdot\rangle \in \mathscr{D}^{\prime}\left(R^{1}\right),
$$

where

$$
\langle s, \psi\rangle=\lim _{\nu \rightarrow \infty} \int_{R^{1}} s(\nu)(x) \psi(x) d x, \quad \forall \psi \in \mathscr{D}\left(R^{1}\right)
$$

One of the basic ideas in the construction of the associative and commutative distribution multiplication in [5] and [6], is the way the weakly convergent sequences of smooth functions representing the Dirac $\delta$ distribution are chosen:

(5) $\mathscr{Z}_{\delta}^{0}$ is the set of $s \in \mathscr{Y}_{0}$, satisfying the conditions:

(5.1) $\langle s, \cdot\rangle=\delta$,

(5.2) $\forall \epsilon>0: \exists \nu_{\epsilon} \in N: \forall \nu \in N$,

$$
\nu \geqq \nu_{\epsilon}, x \in R^{1},|x| \geqq \epsilon: s(\nu)(x)=0
$$

(5.3) $\forall p \in N: \exists \nu_{p} \in N: \forall \nu \in N$,

$$
\nu \geqq \nu_{p}: W(s(\nu), \cdots, s(\nu+p))(0) \neq 0 .
$$

where $W\left(\psi_{1}, \cdots, \psi_{m}\right)(x), x \in R^{l}$, denotes the Wronskian function of $\psi_{1}, \cdots, \psi_{m} \in \mathscr{C}^{\infty}\left(R^{1}\right)$.

The condition (5.3), called "strong local presence of $s$ in $x=0$ " and replaced in [6] by a weaker form, plays a central role in the associative, commutative distribution multiplication presented in [5] and [6].

(6) for $p \in \bar{N}$, denote by $\mathscr{V}_{\delta, p}^{0}$ the set of $v \in \mathscr{V}_{0}$, satisfying the above condition (5.2), as well as

(6.1) $\forall q \in N, q \leqq p: \exists \nu_{q} \in N: \forall \nu \in N: \nu \geqq \nu_{q} \Rightarrow D^{q} v(\nu)(0)=0$;

(7) $\mathscr{S}_{\delta}^{0}=\left\{s \in \mathscr{S}_{0} \mid \operatorname{supp}\langle s, \cdot\rangle \subset\{0\}\right\}$;

(8) $\mathscr{V}_{\delta, p}$, with $p \in \bar{N}$, and $\mathscr{S}_{\delta}$ are the vector subspaces generated in $\mathscr{W}$ by $\cup_{x \in R^{1}} \tau_{x} \mathscr{V}_{\delta, p}^{0}$, respectively $\cup_{x \in R^{1}} \tau_{x} \mathscr{P}_{\delta}^{0}$;

(9) $\mathscr{Z}_{\delta}=X_{x \in R^{2}} \tau_{x} \mathscr{Z}_{\delta}^{0}$;

(10) for $\Sigma=\left(s_{x} \mid x \in R^{1}\right) \in \mathscr{Z}_{\delta}$, denote by $\mathscr{S}(\Sigma)$ the vector subspace generated in $\mathscr{S}_{0}$ by the sequences $D^{p} s_{x}$, with $x \in R^{1}, p \in N$.

And now, the definition of the associative, commutative algebras 
$\left(A_{p, \lambda} \mid p \in \bar{N}, \lambda \in \Lambda\right)$, where $\Lambda$ is the set of all $\lambda=\left(\Sigma, \mathscr{S}_{1}\right)$ with $\Sigma \in \mathscr{Z}_{\delta}$ and $\mathscr{S}_{1}$ vector subspace in $\mathscr{S}_{0}$, such that $\left(U+\mathscr{S}_{\delta}\right) \cap \mathscr{S}_{1}=\mathcal{O}$ and $\mathscr{S}_{0}=$ $\mathscr{U}+\mathscr{S}_{\delta}+\mathscr{S}_{1}$.

Suppose $p \in \bar{N}, \lambda=\left(\Sigma, \mathscr{S}_{1}\right) \in \Lambda$ and denote

(11) $\mathscr{S}_{p, \lambda}=\mathscr{V}_{\delta, p} \oplus \mathcal{U} \oplus \mathscr{S}(\Sigma) \oplus \mathscr{P}_{1}$;

(12) $\mathscr{A}_{p, \lambda}$ the smallest subalgebra in $\mathscr{W}$, containing $\mathscr{S}_{p, \lambda}$ and invariant of the mapping $D: \mathscr{W} \rightarrow \mathscr{W}$;

(13) $\mathscr{I}_{p, \lambda}$ the vector subspace generated in $\mathscr{W}$ by $\mathscr{V}_{\delta, p} \cdot \mathscr{A}_{p, \lambda}$.

Then (see [5] and [6])

(1) $A_{p, \lambda}=\mathscr{A}_{p, \lambda} / \mathscr{I}_{p, \lambda}$,

(2) $D: A_{p+1, \lambda} \rightarrow A_{p, \lambda}$ is given by

$$
D\left(t+\mathscr{I}_{p+1, \lambda}\right)=D t+\mathscr{I}_{p, \lambda}, \quad \forall t \in \mathscr{A}_{p+1, \lambda}
$$

3. Multiplication by $1 / x^{m}, m=1,2, \cdots$ It is shown (see Corollary 2) that in the algebras $A_{p, \lambda}$, the multiplication by $1 / x^{m}$ does not represent the division by $x^{m}$.

Theorem 1. Suppose $T \in A_{p, \lambda}$, with given $p \in \bar{N}, \lambda \in \Lambda$.

Suppose $\psi \in \mathscr{C}^{\infty}\left(R^{1}\right)$ such that for a certain $m \in \bar{N}$

$$
D^{q} \psi(0)=0, \quad \forall q \in N, \quad q \leqq m .
$$

If there exists $\chi \in \mathscr{C}^{\infty}\left(R^{1}\right)$ such that $\psi \cdot T=\chi$ in $A_{p, \lambda}$, then:

$$
D^{q} \chi(0)=0, \quad \forall q \in N, \quad q \leqq \min \{p, m\} .
$$

Proof. Assume $T=t+\mathscr{I}_{p, \lambda}$, with $t \in \mathscr{A}_{p, \lambda}$. Then $\psi \cdot T=\chi$ in $A_{p, \lambda}$ implies $u(\chi)=u(\psi) \cdot t+w$, with $w \in \mathscr{I}_{p, \lambda}$. Therefore,

$$
\forall q \in N, q \leqq p: \exists \nu_{q} \in N: \forall \nu \in N, \nu \geqq \nu_{q}: D^{q} w(\nu)(0)=0 .
$$

Since $\chi=\psi \cdot t(\nu)+w(\nu), \forall \nu \in N$, the proof is completed.

Corollary 1. Suppose $T \in A_{p, \lambda}$, with given $p \in \bar{N}, \lambda \in \Lambda$.

If $\psi \in \mathscr{C}^{\infty}\left(R^{1}\right)$ such that $\psi(0) \neq 0$, then, $x^{m} \cdot T \neq \psi$ in $A_{p, \lambda}, \forall m \in N$, $m \geqq 1$.

Corollary 2. If $m \in N, m \geqq 1$, then, $x^{m} \cdot\left(1 / x^{m}\right) \neq 1$, in each of the algebras $A_{p, \lambda}, p \in \bar{N}, \lambda \in \Lambda$.

4. Division by $x^{m}, m=1,2, \cdots$. First, in Theorem 2, a 
sufficient condition is given for $T \in A_{p, \lambda}$, in order to be a solution of the equation $x^{m} \cdot T=0 \in A_{p, \lambda}$, where $m \in N, m \geqq 1$.

For $p \in \bar{N}$ and $\lambda \in \Lambda$, denote by $B_{p, \lambda}^{0}$ all the elements $T \in A_{p, \lambda}$ of the form $T=t+\mathscr{I}_{p, \lambda}$, where $t \in \mathscr{A}_{p, \lambda} \cap \mathscr{V}_{0}$ and satisfies also (5.2) in $\S 2$.

Proposition 1. Suppose given $p \in \bar{N}, \lambda \in \Lambda$ and $\psi \in \mathscr{C}^{\infty}\left(R^{1}\right)$, such that, for a certain $q \in \bar{N}, q \geqq p$ :

$$
D^{r} \psi(0)=0, \quad \forall r \in N, \quad r \leqq q .
$$

Then, $\psi \cdot B_{p, \lambda}^{0}=\{0\} \subset A_{p, \lambda}$.

Proof. Assume $T \in B_{p, \lambda}^{0}$ and $T=t+\mathscr{I}_{p, \lambda}$, with $t \in \mathscr{A}_{p, \lambda} \cap \mathscr{V}_{0}$ and satisfying (5.2) in $\S 2$. Then, $\psi \cdot T=u(\psi) \cdot t+\mathscr{I}_{p . \lambda}$. But, obviously, $u(\psi) \cdot t \in \mathscr{V}_{\delta, q}^{0} \subset \mathscr{V}_{\delta, p}^{0} \subset \mathscr{I}_{p, \lambda}$, hence, $T=0 \in A_{p, \lambda}$.

Theorem 2. Suppose given $p \in N, \lambda \in \Lambda$ and $m \in N, m \geqq 1$.

Then, any

$$
T_{0}=\sum_{0 \leqq i \leqq k} x^{r_{1}} \cdot T_{1 \mathrm{t}} \cdot T_{2 \mathrm{t}}+\sum_{0 \leqq I \leqq h} x^{q_{1}} \cdot D^{p_{1}} \delta \cdot T_{3,}
$$

with $k, h, r_{i}, q_{l}, p_{l} \in N, r_{i}>p-m$, $q_{1}>\max \left\{p, p_{\jmath}\right\}-m$, and $T_{11} \in B_{p, \lambda}^{0}, T_{2,}, T_{3,} \in A_{p, \lambda}$, will be a solution in $A_{p, \lambda}$ of the equation $x^{m} \cdot T=0$.

Proof. According to Proposition 1, $x^{m} \cdot x^{r_{1}} \cdot T_{11}=x^{m+r_{1}} \cdot T_{14}=$ $0 \in A_{p, \lambda}$, since $m+r_{\imath}>p$. According to (4) in $\S 1$ (see also 3) in Theorem $6, \quad \S 8 \quad[5]), \quad x^{m} \cdot x^{q_{1}} \cdot D^{p_{1}} \delta=x^{m+q_{1}} \cdot D^{p_{1}} \delta=0 \in A_{p_{1} \lambda}$, since $m+q_{l}>$ $\max \left\{p, p_{j}\right\}$.

It results the following sufficient condition on $T \in A_{p, \lambda}$, solution of the equation $x^{m} \cdot T=S \in A_{p, \lambda}$.

Corollary 3. Suppose $S \in A_{p, \lambda}$, with $p \in N, \lambda \in \Lambda$ given and $m \in N, m \geqq 1$.

If $T_{1}$ is any solution in $A_{p, \lambda}$ of the equation $x^{m} \cdot T=S$ and $T_{0}$ is given as in Theorem 2, then $T=T_{1}+T_{0}$ will be again a solution of that equation.

Before a necessary condition is given on $T \in A_{p, \lambda}$, solution of the equation $x^{m} \cdot T=0 \in A_{p, \lambda}$, the notion of support of the elements in $A_{p, \lambda}$ will be defined. 
Suppose $T \in A_{p, \lambda}$, with $p \in \bar{N}, \lambda \in \Lambda$ given and $E \subset R^{1}$. Then,

(1) $T$ vanishes on $E$, only if $T=t+\mathscr{I}_{p, \lambda}$, with $t \in \mathscr{A}_{p, \lambda}$, such that $t(\nu)(x)=0, \forall \nu \in N, \nu \geqq \nu_{0}, x \in E$.

(2) $T$ strictly vanishes on $E$, only if $T$ vanishes on a certain open set $G \subset R^{1}$, containing $E$.

(3) $T$ is supported by $E$, only if for every open set $G \subset R^{1}$, containing $E$, one can write $T=t+\mathscr{I}_{p, \lambda}$, with $t \in \mathscr{A}_{p, \lambda}$, such that $\operatorname{supp} t(\nu) \subset G, \forall \nu \in N, \nu \geqq \nu_{0}$.

The support of $T$ is defined as the closed set

$$
\operatorname{supp} T=R^{1} \backslash\left\{x \in R^{1} \mid T \text { strictly vanishes on }\{x\}\right\} \text {. }
$$

Obviously, for the distributions in $\mathscr{C}^{\infty}\left(R^{1}\right) \bigoplus \mathscr{D}_{\delta}^{\prime}\left(R^{1}\right)$, the above notion of support is identical with the usual one for distributions.

Proposition 2. Suppose $x_{0} \in R^{1}$ and $q \in N$, then, $D^{q} \delta_{x_{0}} \in A_{p, \lambda}$, for $p \in \bar{N}, \lambda \in \Lambda$, and

(1) $D^{q} \delta_{x_{0}}$ is supported by $\left\{x_{0}\right\}$ and $\operatorname{supp} D^{a} \delta_{x_{0}}=\left\{x_{0}\right\}$,

(2) if $E \subset R^{1}$ and $x_{0} \notin$ closure $E$, then $D^{a} \delta_{x_{0}}$ strictly vanishes on $E$,

(3) $D^{a} \delta_{x_{0}}$ does not vanish on $R^{1} \backslash\left\{x_{0}\right\}$,

(4) $D^{a} \delta_{x_{0}}$ does not vanish on $\left\{x_{0}\right\}$.

Proof. (1), (2) and (3) follow easily.

(4) Assume $\lambda=\left(\Sigma, \mathscr{S}_{1}\right)$ and $\Sigma=\left(s_{x} \mid x \in R^{1}\right)$, then, $D^{q} \delta_{x_{0}}=D^{q} S_{x_{0}}+\mathscr{I}_{p, \lambda}$ and $s_{x_{0}} \in \tau_{x_{0}} \mathscr{Z}_{\delta}^{0}$. Suppose, $D^{a} \delta_{x_{0}}$ vanishes on $\left\{x_{0}\right\}$, then, there exists $t \in \mathscr{A}_{p, \lambda}$, such that $t-D^{q} S_{x_{0}} \in \mathscr{I}_{p, \lambda}$ and $t(\nu)\left(x_{0}\right)=0, \quad \forall \nu \in N, \quad \nu \geqq$ $\nu_{0}$. Denoting $v=t-D^{q} S_{x_{0}}$, the relation $v \in \mathscr{I}_{p, \lambda}$ implies $\nu(\nu)\left(x_{0}\right)=0$, $\forall \nu \in N, \nu \geqq \nu_{1}$. Therefore, it results

$$
D^{q} S_{x_{0}}(\nu)\left(x_{0}\right)=t(\nu)\left(x_{0}\right)-v(\nu)\left(x_{0}\right)=0, \quad \forall \nu \in N, \quad \nu \geqq \nu_{2} .
$$

But, that relation implies $W\left(s_{x_{0}}(\nu), \cdots, s_{x_{0}}(\nu+q)\right)\left(x_{0}\right)=0, \forall \nu \in N, \nu \geqq$ $\nu_{2}$, which contradicts the assumption $s_{x_{0}} \in \tau_{x_{0}} \mathscr{Z}_{\delta}^{0}$.

Remark. The property of the Dirac distributions that $D^{a} \delta_{x_{1}}$ does not vanish on $\left\{x_{0}\right\}, \forall x_{0} \in R^{1}, q \in N$, is a direct consequence of the "condition of strong local presence" (see (5.3) in \$2) and it is proper for the distribution multiplication presented in [5] and [6]. The "delta sequences" generally used (see [2]) do not necessarily prevent the vanishing of $D^{a} \delta_{x_{0}}$ on $\left\{x_{0}\right\}$.

Theorem 3. Suppose $T \in A_{p, \lambda}$ with $p \in \bar{N}, \lambda \in \Lambda$ given. 
If $x^{m} \cdot T=0 \in A_{p, \lambda}$, for a certain $m \in N, m \geqq 1$, then $T$ is supported by $\{0\}$, hence supp $T \subset\{0\}$.

Proof. Assume $T=t+\mathscr{I}_{p, \lambda}$, with $t \in \mathscr{A}_{p, \lambda}$. Then $x^{m} \cdot T=0 \in A_{p, \lambda}$ implies $u\left(x^{m}\right) \cdot t \in \mathscr{I}_{p, \lambda}$, therefore, according to the definition of $\mathscr{I}_{p, \lambda}$ (see (13), §2), it results

$$
u\left(x^{m}\right) \cdot t=\sum_{0 \leqq ı \leqq k} v_{l} \cdot a_{l}
$$

with $k \in N, v_{\imath} \in \mathscr{V}_{\delta, p}, a_{\imath} \in \mathscr{A}_{p, \lambda}$.

Now, due to the definition $\mathscr{V}_{\delta, p}$ (see (8) and (6), §2), it follows that: $\forall i \in\{0, \cdots, k\}: \exists X_{t} \subset R^{1}, X_{t}$ finite: $v_{t}=\Sigma_{x \in X_{i}} v_{t x}$, where $v_{t x} \in \tau_{x} \mathscr{V}_{\delta, p}^{0}$.

Concluding, there exists $X \subset R^{1}, X$ finite, such that

$$
u\left(x^{m}\right) \cdot t=\sum_{x \in X} \sum_{0 \leqq j \leqq h} v_{x j} \cdot b_{x j} \quad \text { with } \quad h \in N, \quad v_{x j} \in \tau_{x} \mathscr{V}_{\delta, p}^{0}, \quad b_{x j} \in \mathscr{A}_{p, \lambda}
$$

It will be shown now, that in the above relation, one can consider $X=\{0\}$. Indeed, suppose $x_{0} \in X \backslash\{0\}$, then $v_{x_{0 j}} \in \tau_{x_{0}} \mathscr{V}_{\delta, p}^{0}$ with $0 \leqq j \leqq$ $h$. The condition (5.2) in $\$ 2$, results in the existence of $w_{x_{0 j} j} \in \mathcal{W}$, with $0 \leqq j \leqq h$, such that $v_{x^{\prime \prime j}}(\nu)(x)=x^{m} \cdot w_{x_{0}}(\nu)(x), \quad \forall 0 \leqq j \leqq h, x \in R^{1}$, $\nu \in N, \nu \geqq \nu_{0}$. Moreover, $w_{x_{1}, j} \in \tau_{x_{0}} \mathscr{V}_{\delta, p}^{0}, \forall 0 \leqq j \leqq h$, since $v_{x_{0 j}} \in \tau_{x_{0}} \mathscr{V}_{\delta, p}^{0}$, with $0 \leqq j \leqq h$, and $x_{0} \neq 0$.

Denoting

$$
v=\sum_{\substack{x_{0} \in X \\ x_{0} \neq 0}} \sum_{0 \leqq J \leqq h} w_{x_{0 I J}} \cdot b_{x_{0 \prime}}
$$

it results $v \in \mathscr{I}_{p, \lambda}$, hence, $T=t_{1}+\mathscr{I}_{p, \lambda}$, where $t_{1}=t-v \in \mathscr{A}_{p, \lambda}$. But $u\left(x^{m}\right) \cdot t_{1}=u\left(x^{m}\right) \cdot t-u\left(x^{m}\right) \cdot v=\sum_{0 \leqq I \leqq h} v_{0,} \cdot b_{0,}$.

Since $v_{0,}$, with $0 \leqq j \leqq h$, satisfy (5.2) in $\S 2$, it follows that $u\left(x^{m}\right) \cdot t_{1}$ and, therefore $t_{1}$ satisfy the same condition. Thus, $T=t_{1}+\mathscr{I}_{p, \lambda}$ is supported by $\{0\}$, which obviously results in supp $T \subset\{0\}$.

\section{REFERENCES}

1. H. Kang, J. Richards, A general definition of convolution for distributions, (to appear).

2. J. Mikusinski, On the square of the Dirac delta distribution, Bull. Acad. Pol. Sci., 14, 9, (1966), 511-513.

3. E. Rosinger, Embedding the $\mathscr{D}^{\prime}\left(R^{n}\right)$ distributions in pseudotopological algebras, Stud. Cerc. Mat., 18. 5, (1966), 687-729.

4. - Pseudotopological spaces. Embedding the $\mathscr{D}^{\prime}\left(R^{n}\right)$ distributions into algebras, Stud. Cerc. Mat., 20, 4, (1968), 553-582. 
5. - A distribution multiplication theory, Haifa Technion's Preprint Series, AMT-31, October 1974 (to appear).

6. - An associative, commutative distribution multiplication, Technical Report, Haifa Technion, March 1976 (to appear).

7. L. Schwartz, Sur l'impossibilité de la multiplication des distributions, C. R. Acad. Sci. Paris, 239 (1954), 847-848.

Received June 23, 1975 and in revised form April 12, 1976.

Technion - IsRael Institute of Technology 




\section{Pacific Journal of Mathematics}

\section{Vol. 66, No. $1 \quad$ November, 1976}

Helen Elizabeth. Adams, Factorization-prime ideals in integral domains ............ Patrick Robert Ahern and Robert Bruce Schneider, The boundary behavior of Henkin's kernel.

Daniel D. Anderson, Jacob R. Matijevic and Warren Douglas Nichols, The Krull

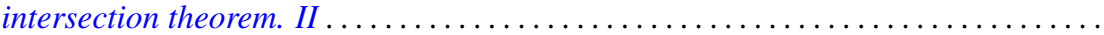

Efraim Pacillas Armendariz, On semiprime P.I.-algebras over commutative regular

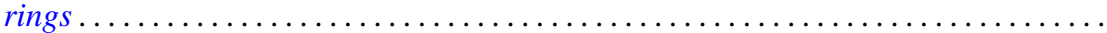

Robert H. Bird and Charles John Parry, Integral bases for bicyclic biquadratic fields

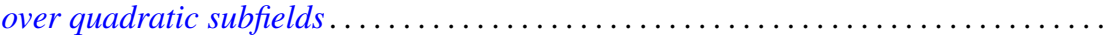

Tae Ho Choe and Young Hee Hong, Extensions of completely regular ordered

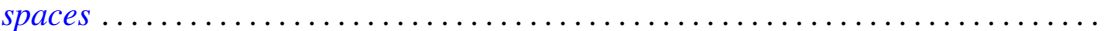

John Dauns, Generalized monoform and quasi injective modules ...............

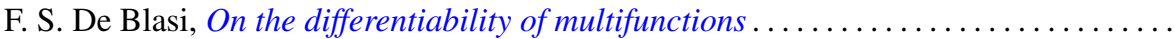

Paul M. Eakin, Jr. and Avinash Madhav Sathaye, R-endomorphisms of $R[[X]]$ are

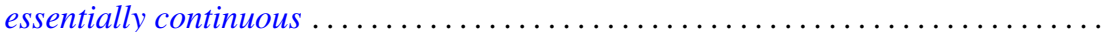

Larry Quin Eifler, Open mapping theorems for probability measures on metric

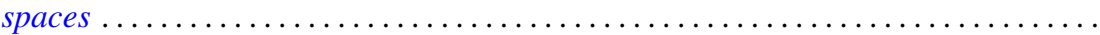

Garret J. Etgen and James Pawlowski, Oscillation criteria for second order self adjoint

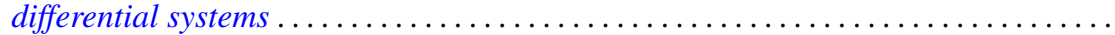

Ronald Fintushel, Local $S^{1}$ actions on 3-manifolds .

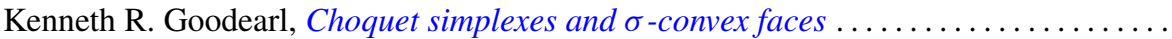

John R. Graef, Some nonoscillation criteria for higher order nonlinear differential

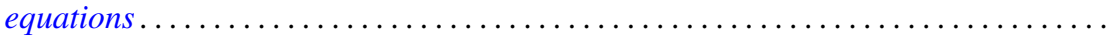

Charles Henry Heiberg, Norms of powers of absolutely convergent Fourier series: an example.

Les Andrew Karlovitz, Existence of fixed points of nonexpansive mappings in a space

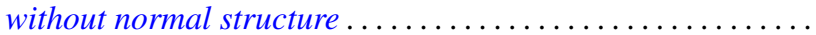

Gangaram S. Ladde, Systems of functional differential inequalities and functional

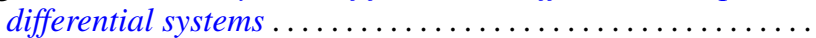

Joseph Michael Lambert, Conditions for simultaneous approximation and interpolation with norm preservation in $C[a, b]$.

Ernest Paul Lane, Insertion of a continuous function.

Robert F. Lax, Weierstrass points of products of Riemann surfaces .

Dan McCord, An estimate of the Nielsen number and an example concerning the Lefschetz fixed point theorem...

Paul Milnes and John Sydney Pym, Counterexample in the theory of continuous functions on topological groups...

Peter Johanna I. M. De Paepe, Homomorphism spaces of algebras of holomorphic functions

Judith Ann Palagallo, A representation of additive functionals on $L^{p}$-spaces,

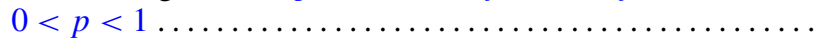

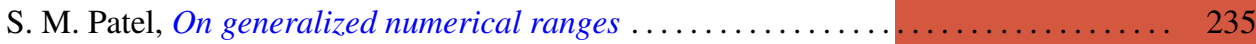

Thomas Thornton Read, A limit-point criterion for expressions with oscillatory

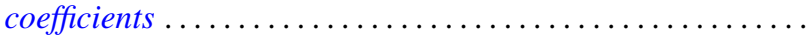

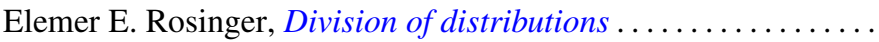

Peter S. Shoenfeld, Highly proximal and generalized almost finite

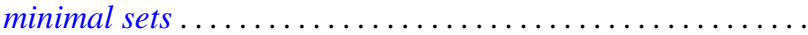

R. Sirois-Dumais and Stephen Willard, Quotient-universal sequential spaces

Robert Charles Thompson, Convex and concave functions of singular values of matrix sums....

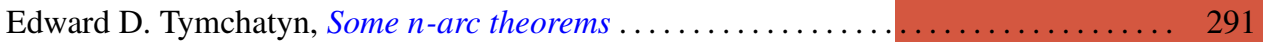

